Marquette University

e-Publications@Marquette

Biomedical Engineering Faculty Research and

Publications

Biomedical Engineering, Department of

$12-2012$

\title{
Preliminary Investigation of Residual Limb Plantarflexion and Dorsiflexion Muscle Activity During Treadmill Walking for Trans- tibial Amputees
}

\author{
M. Barbara Silver-Thorn \\ Marquette University, barbara.silver-thorn@marquette.edu \\ Thomas Current \\ Hanger Prosthetics and Orthotics \\ Benjamin Kuhse \\ Hanger Prosthetics and Orthotics
}

Follow this and additional works at: https://epublications.marquette.edu/bioengin_fac

Part of the Biomedical Engineering and Bioengineering Commons

\section{Recommended Citation}

Silver-Thorn, M. Barbara; Current, Thomas; and Kuhse, Benjamin, "Preliminary Investigation of Residual Limb Plantarflexion and Dorsiflexion Muscle Activity During Treadmill Walking for Trans-tibial Amputees" (2012). Biomedical Engineering Faculty Research and Publications. 58.

https://epublications.marquette.edu/bioengin_fac/58 
Marquette University

e-Publications@Marquette

\section{Biomedical Engineering Faculty Research and Publications/College of} Engineering

This paper is NOT THE PUBLISHED VERSION; but the author's final, peer-reviewed manuscript. The published version may be accessed by following the link in the citation below.

Prosthetics and Orthotics International, Vol. 36, No. 4 (December 1, 2012): 435-442. DOI. This article is (C) SAGE Publications and permission has been granted for this version to appear in $\underline{\mathrm{e}-}$ Publications@Marquette. SAGE Publications does not grant permission for this article to be further copied/distributed or hosted elsewhere without the express permission from SAGE Publications.

\section{Preliminary Investigation of Residual Limb Plantarflexion and Dorsiflexion Muscle Activity During Treadmill Walking for Trans- Tibial Amputees}

Barbara Silver-Thorn

Marquette University, Milwaukee, WI

Thomas Current

Marquette University, Milwaukee, WI

Benjamin Kuhse

Hanger Prosthetics and Orthotics, Milwaukee, WI 


\begin{abstract}
Background:

Novel powered prosthetic ankles currently incorporate finite state control, using kinematic and kinetic sensors to differentiate stance and swing phases/sub-phases and control joint impedance and position or torque. For more intuitive control, myoelectric control of the ankle using the remnant residual limb dorsiflexors and plantarflexors, perhaps in concert with kinetic and kinematic sensors, may be possible.
\end{abstract}

\title{
Objective:
}

The specific research objective was to assess the feasibility of using myoelectric control of future active or powered prosthetic ankle joints for trans-tibial amputees.

\section{Study Design:}

The project involved human subject trials to determine whether current techniques of myoelectric control of upper extremity prostheses might be readily adapted for lower extremity prosthetic control.

\section{Methods:}

Gait analysis was conducted for three unilateral trans-tibial amputee subjects during ambulation on an instrumented split belt treadmill. Data included ankle plantarflexor and dorsiflexor activity for the residual limb, as well as lower limb kinematics and ground reaction forces and moments of both the sound and prosthetic limbs.

\section{Results:}

These data indicate that: 1 ) trans-tibial amputees retain some independent ankle plantarflexor and dorsiflexor muscle activity of their residual limb; 2 ) it is possible to position surface electromyographic electrodes within a trans-tibial socket that maintain contact during ambulation; 3 ) both the plantarflexors and dorsiflexors of the residual limb are active during gait; 4) plantarflexor and dorsiflexor activity is consistent during multiple gait cycles; and 5) with minimal training, trans-tibial amputees may be able to activate their plantarflexors during push-off.

\section{Conclusions:}

These observations demonstrate the potential for future myoelectric control of active prosthetic ankles.

\section{Clinical relevance}

This study demonstrated the feasibility of applying upper extremity prosthetic myoelectric signal acquisition, processing and control techniques to future myoelectric control of active prosthetic ankles for trans-tibial amputees.

\section{Keywords}

Biomechanics of prosthetic/orthotic devices, gait analysis, prosthetic design, rehabilitation of amputees

\section{Background}

Several recent prosthetic design studies ${ }^{1-5}$ have focused on the development of powered ankles to assist lower limb amputees with push-off, attempting to decrease energy costs associated with ambulation. Designing a powered prosthetic ankle of similar size and mass as that of the intact ankle that provides sufficient torque to propel an amputee is particularly challenging. In addition, there is 'no clear control target or "gold standard" for the prosthesis to be controlled, against which to gauge the effectiveness' ${ }^{4}$ 
In contrast to upper extremity function during various activities of daily living, gait is a cyclic activity that has often been controlled both prosthetically and orthotically using finite state control. ${ }^{1}$ As such, these novel powered prosthetic ankles (and knees) incorporate finite state control. Various kinematic (ankle position, velocity and/or acceleration) and kinetic (ankle torque, plantar and/or pylon loading) sensors and threshold levels have been used to differentiate stance and swing phases/sub-phases and control joint impedance (stiffness, damping) and position or torque. ${ }^{1-3,6}$ These finite state control algorithms have demonstrated comparable prosthetic versus intact ankle torque during level walking. However, the use of interactive extrinsic control incorporating residual limb electromyography (EMG) or neural integration has been advocated for active ankle (and active knee) control for other types of ambulation and activities of daily living, as well as for the transitions between such tasks. ${ }^{7}$

Au et al. ${ }^{8}$ explored the use of surface EMG (biomimetic muscle model and neural network approaches) to control a simulated ankle trajectory. While the biomimetic EMG-controller demonstrated a smoother and more natural movement pattern compared with the neural network approach, this controller was not incorporated in their active ankle design. Instead Au et al. ${ }^{9}$ used a myoelectric processing unit to detect an amputee's intent, using the variance in tibialis anterior and gastrocnemius muscle activity to set the desired ankle position and transition to and from level-ground walking and stair descent, respectively.

The control of externally powered upper extremity prosthetic components routinely incorporate myoelectric control, which have been used in conjunction with switches (rocker switches, force sensitive resistors (FSRs), linear variable resistors, etc.). Details regarding the preparation of an upper extremity amputee for a myoelectric device, the EMG acquisition and signal processing, and EMG feature extraction are summarized in Zecca. ${ }^{10}$ Most commercial prosthetic myoprocessors are based on one dimension of the EMG signal: variance or mean absolute value. ${ }^{10}$ Myoelectric control of upper extremity prostheses typically involves single- or dual-site control to control the speed or grip force of terminal device prehension in a constant or proportional fashion. The prosthetist uses a myotester to evaluate appropriate sites for electrode placement to control a terminal device or powered elbow, and to assess the individual's ability to independently control the muscle pairs, producing muscle signals with minimal co-contraction and varying amplitude. If successful, the externally powered terminal device can then be controlled using either on-off control (e.g. terminal device opens/closes at a set velocity when the muscle signal exceeds a specific threshold) or proportional control such that the speed at which the terminal device is opened (or closed) is proportional to the muscle signal amplitude. If the individual is unable to independently contract their remnant flexors and extensors, single site control may be used such that the level of contraction dictates motor direction (moderate contraction=closing, intense contraction=opening, no activity=motor off).

Early designs of powered lower limb prostheses attempted to use myoelectric control. As gait is a cyclic activity, these designs $s^{4,11}$ used EMG activity of the sound limb, echoing this signal to control the prosthetic limb. Studies investigating EMG activity of the lower extremity residual limb have focused on the intact muscles of the hip and knee, muscles that are not affected by amputation surgery and that can be monitored using electrodes outside the prosthetic socket. ${ }^{12-15}$ For more intuitive control of ankle position, stiffness, damping and/or torque in active prosthetic ankles, myoelectric control of the ankle using the remnant residual limb dorsiflexors and plantarflexors, perhaps in concert with kinetic and kinematic sensors, may be possible. Analogous to myoelectric control of terminal device prehension, the future feasibility of on-off (e.g. prosthetic ankle plantarflexes at a set velocity when the plantarflexor muscle signal exceeds a specific threshold) or proportional (e.g. speed or prosthetic ankle plantarflexion torque is proportional to the plantarflexion muscle signal amplitude) control of an active prosthetic ankle might then be investigated. More complex control algorithms integrating ankle kinematic and kinetic sensors to control ankle position, stiffness, damping and/or power might also be investigated. 
While the clinical motivation for this study is future myoelectric control of lower extremity prostheses, particularly active prosthetic ankle components, the purpose of this study and manuscript is to investigate: 1) whether EMG activity of the remnant ankle dorsiflexors and plantarflexors remains after transtibial amputation; 2) whether these EMG signals can be acquired dynamically within a prosthetic socket using commercially available upper extremity prosthetic technology; 3) whether such EMG activity is similar to that observed preamputation during gait; and 4) whether subjects can modify this EMG activity so as to increase plantarflexion activity during late stance.

\section{Methods}

Three unilateral trans-tibial amputees were recruited for this study (Table 1). Subject selection criteria included: the ability to walk for at least $100 \mathrm{~m}$ without the use of an assistive device, no prior history of falls or dermatological problems of the residual limb tissues, stable residual limb volume, and prosthetic user (endoskeletal prosthesis) for at least six months. Subjects were recruited from client databases of collaborating prosthetists (Current and Kuhse).

Table 1. Subject characteristics and definitive prostheses.

\begin{tabular}{|l|l|l|l|}
\hline Subject & A1 & A2 & A3 \\
\hline Age $(\mathrm{yrs})$ & 63 & 37 & 49 \\
\hline Time since amputation (yrs) & 4 & 1.8 & 2 \\
\hline Cause of amputation & Trauma & Trauma & Trauma \\
\hline Amputated side & R & L & R \\
\hline Weight $(\mathrm{kg})$ & 132.4 & 66.7 & 99.8 \\
\hline Height $(\mathrm{cm})$ & 177.8 & 170.2 & 182.9 \\
\hline Current prosthetic foot & BioQuest & Axia & Soleus \\
\hline Current suspension & $\begin{array}{l}\text { Alpha Locking } \\
\text { Liner }\end{array}$ & $\begin{array}{l}\text { IceRoss Locking } \\
\text { Liner }\end{array}$ & $\begin{array}{l}\text { PSI liner with elevated } \\
\text { vacuum }\end{array}$ \\
\hline $\begin{array}{l}\text { Additional prosthetic } \\
\text { components }\end{array}$ & n/a & DuraShock pylon & Otto Bock 4R40 rotator \\
\hline Socket type & TSB & TSB & TSB \\
\hline
\end{tabular}

Testing consisted of potential myosite identification, experimental socket fabrication with myosite verification, and gait analysis. This protocol was reviewed and approved by the Institutional Review Board at IRB at Marquette University; written informed consent was solicited for each subject prior to research participation.

Simple myotesting was conducted using the MyoBoy (model \#757M11, Otto Bock, Duderstadt, Germany), a myotester currently used by prosthetists for upper extremity myoelectric control evaluation, training, and fitting, to assess each subject's potential for two-site control of the dorsiflexors and plantarflexors of the residual limb. After removing their prosthesis, the subject was asked to 'lift' and 'point' the toes of their phantom limb. The prosthetist observed and palpated the subject's residual limb during these tests, identifying potential dorsiflexor and plantarflexor site locations. The prosthetist then placed the MyoBoy ${ }^{\circ}$ electrodes over these potential sites, identifying sites at which the subject was able to elicit independent muscle activity. These optimal electrode sites on the residual limb were marked with a wax pencil. The amputee donned a gel liner thereby transferring the electrode site locations to the liner interior. The prosthetist punched button electrodes (model \#EL-02, $1 \mathrm{~cm}$ dia, LTI: Liberating Technologies, Inc., Holliston, MA) through the gel liner at these locations (two electrodes along longitudinal axis of this site, along the muscle belly; a third ground electrode was positioned such that it completed an equilateral triangle). Electrode pre-amplifiers (model \#DC125, LTI) and cables $(30 \mathrm{~cm})$ were then connected to these button electrodes. The amputee donned the liner with electrodes, 
taking care to orient the liner in a consistent manner such that the electrodes were positioned over the desired test sites. These electrodes were then connected to the MyoBoy to verify electrode placement and muscle signal. If necessary, the electrode sites or liner donning was modified, and the pre-amplifier gains were adjusted.

A thermolyn plastic check socket that duplicated the subject's current socket was then fabricated, inclusive of connective hardware; reliefs and 'channels' were added to accommodate the button electrodes and their screw posts as the socket was donned and loaded. The check socket was attached to the distal pylon and foot of the subject's current endoskeletal prosthesis. This experimental prosthesis was then donned (sitting, standing, walking in parallel bars) to ensure that the subject did not experience discomfort. The socket was modified as needed by local heating to manipulate the socket shape. The electrodes were then connected to the MyoBoy ${ }^{\oplus}$ to ensure that site location (and comfort) was maintained during walking; the pre-amplifier gains were adjusted as needed. The amputee then resumed use of their current liner; the prosthetist replaced the experimental socket with the subject's original socket.

Gait analysis was conducted in the Locomotor Neurorehabilitation Laboratory at Marquette University. Prior to gait analysis, the amputee removed their current prosthesis and the prosthetist replaced the socket with the experimental socket. The subject donned the new gel liner with button electrodes and the experimental prosthesis. The electrode sites and muscle signals were verified, adjusting the pre-amplifier gains as necessary. Anthropometric measures (height, weight, ankle width (between medial and lateral malleoli), knee width (between medial and lateral femoral condyles, lower extremity limb length (hip to medial malleoli), and sole incline of shoe) were then taken. Reflective markers were placed on both lower extremities (toe, lateral malleoli, heel, mid-shank, lateral femoral epicondyle, lateral thigh, anterior superior iliac spine) and the sacrum; the marker locations on the prosthetic limb were approximated based on the sound limb locations. The subject stood on a treadmill; the safety harness was then donned and secured (Figure 1). Kinetic and kinematic data were acquired during level walking using an instrumented split-belt treadmill (Bertec, Columbus, $\mathrm{OH}$ ) and sixcamera 3D motion capture system (Vicon, Oxford, UK). The processed EMG data (LTI EL-02 electrodes and DC125 pre-amplifiers, incorporating differential amplification (high CMRR $>100 \mathrm{~dB}$ ), rectification, band pass (approximately $10-500 \mathrm{~Hz}$ ) and notch $\left(60 \mathrm{~Hz}\right.$ ) filtering ${ }^{10}$ ) were sent to both the MyoBoy (real-time display) and Vicon (synchronous acquisition with kinematic and kinetic data using Vicon Nexus software) using a custom fabricated cable. Kinematic data were acquired at $100 \mathrm{~Hz}$; kinetic and EMG data were acquired at $1000 \mathrm{~Hz}$.

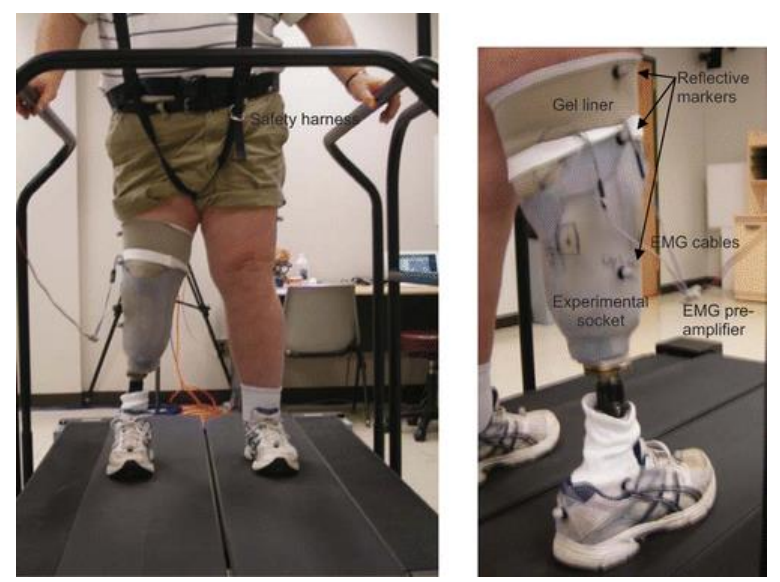

Figure 1. Subject standing in safety harness on split belt treadmill.

The subject slowly accelerated to their self-selected walking speed; subjects were permitted to use the handrails during ambulation if desired. Ten-second trials of kinematic, kinetic and EMG data were collected over a twominute period of quiet walking. The subject was then asked to walk such that they 'pushed off' with their prosthetic foot during late stance; 10 -second trials of kinematic, kinetic and EMG data were collected over a 
second two-minute walking trial. For reference, EMG data corresponding to maximum voluntary contraction of the dorsiflexors and plantarflexors were also acquired.

The kinematic data were processed using Vicon Nexus (v1.4.116) and the PlugInGait model. The resultant c3d files included synchronous lower limb joint angles and ground reaction forces and moments for both the prosthetic and sound limbs, and ankle dorsiflexion and plantarflexion muscle activity of the residual limb. Gait events (heel strike and toe off) were identified based on vertical force magnitude (e.g. threshold set to $2 \%$ body weight). These heel strike and toe off events were used to define the gait cycle (period between successive heel strikes of the prosthetic limb), stance (ipsilateral heel strike to ipsilateral toe off) and swing (ipsilateral toe off to ipsilateral heel strike) periods. The gait cycles were further divided into four stance and three swing periods, as defined by Perry. ${ }^{16}$ These stance periods of the amputated and sound limbs were defined as:

- loading response: ipsilateral heel strike through contralateral toe off

- mid-stance: mid-point between contralateral toe off of loading response and contralateral heel strike of terminal stance

- terminal stance: through contralateral limb strike

- pre-swing: contralateral heel strike through ipsilateral toe off.

The swing phase was divided into thirds: initial swing, mid-swing and terminal swing periods.

The kinetic ground reaction forces and moments were reviewed during these functional phases for each gait cycle. The dorsiflexion and plantarflexion EMG data were reviewed using threshold detection techniques to identify periods in which the dorsiflexors and plantarflexors were active ('on') and quiet ('off'). These EMG data, both the linear envelope or integrated EMG (IEMG) and the corresponding on-off format, were then plotted as a function of gait cycle.

\section{Results}

Kinematic, kinetic and EMG data were acquired during level treadmill walking for three trans-tibial amputees. As the research objective for this study was to determine whether current techniques for myoelectric control of upper extremity prostheses might be readily adapted to lower extremity control, only the EMG data are presented here. Sample EMG data are shown in Figure 2, illustrating the linear envelope IEMG and corresponding on-off periods based on a simple threshold detection algorithm. Full EMG data during nominal treadmill ambulation at the subject's self-selected walking speed are plotted as a function of gait cycle in Figure 3a; the corresponding mean data are shown in Figure 3b. Mean EMG data contrasting nominal versus 'push-off' (e.g. subject was instructed to 'push off' with their prosthetic foot during late stance) walking trials are contrasted in Figure 4 for each of the three test subjects.

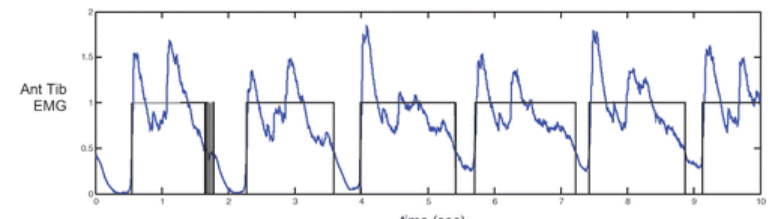

time (sec)

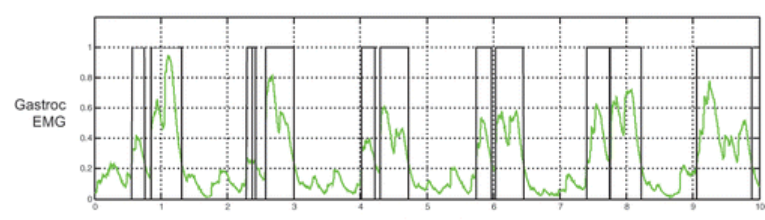

time (sec) 
Figure 2. Sample electromyographic (EMG) data during a nominal walking trial for subject $A 1$. The linear envelope of the dorsiflexor (top) and plantarflexor (bottom) activity is shown, along with the corresponding 'onoff' activity, as assessed with threshold detection (25\% peak EMG amplitude); active periods were set to unity, inactive periods were set to zero.
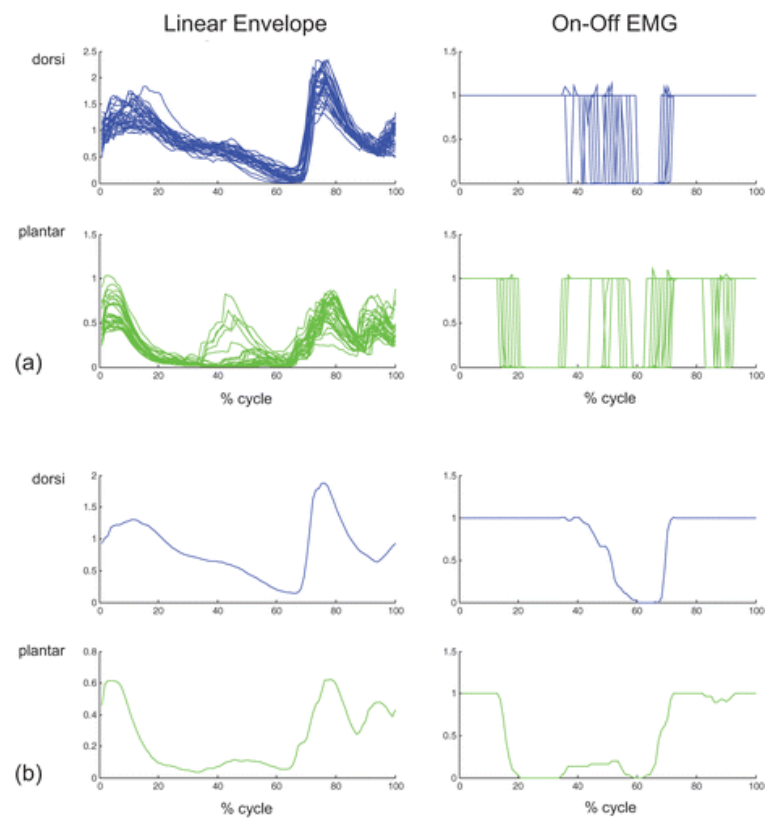

Figure 3. Representative electromyographic (EMG) data for subject A1 during level walking (with handrail support) at the subject's self-selected walking speed: (a) 29 individual gait cycles, (b) corresponding mean data. The graphs include the linear envelope of the EMG (left), and the corresponding 'on-off' activity (right) as assessed with threshold detection.
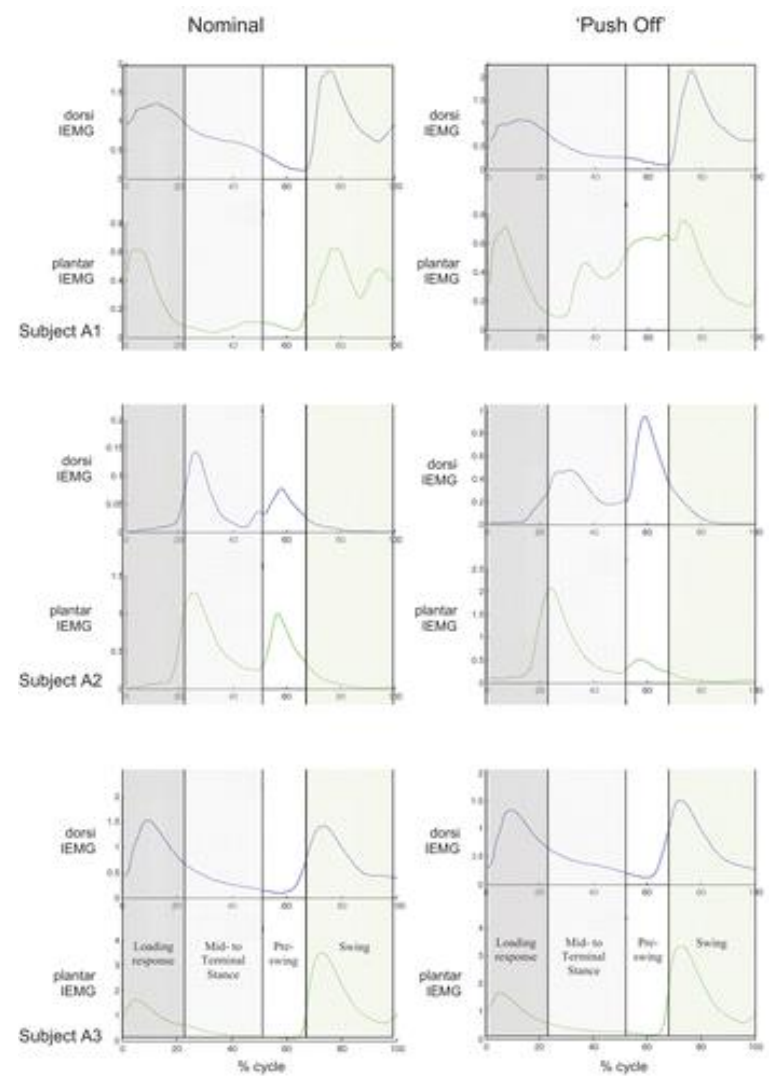
Figure 4. Mean electromyographic (EMG) data for each subject during nominal (left) and 'push off' (right) walking trials. (A1: with handrails, 29-30 gait cycles; A2: with handrails, 38-41 gait cycles; A3: without handrails, 72 gait cycles).

The corresponding kinematic and kinetic data will be used in future studies investigating various integrated control options incorporating EMG, kinematic and/or kinetic data for active ankle-foot prostheses.

\section{Discussion}

Four traumatic trans-tibial amputees were recruited for this preliminary study, although only three subjects opted to participate in gait analysis. Each of these trans-tibial amputees (1.8 to 4 years post-amputation) retained independent control of ankle dorsiflexors and plantarflexors, although the respective muscle sites were altered due to amputation surgery.

Insertion of button electrodes through a gel liner in concert with a prosthetic socket with electrode relief channels resulted in clean, consistent EMG signals with little movement artifact over multiple gait cycles, as seen by linear enveloped IEMG data in Figure 3a. These consistent linear-enveloped IEMG data also indicate that electrode-skin contact was maintained during the treadmill walking trials. The jitter observed in the simplistic on-off EMG data can likely be minimized by incorporation of further signal processing.

For healthy normal subjects, the dorsiflexors are active during loading response to prevent foot slap and during swing to assist with foot clearance; the plantarflexors are active during mid-and terminal-stance to stabilize the foot and provide push off. ${ }^{17}$ These trans-tibial amputees continued to activate their ankle dorsiflexors and plantarflexors during ambulation (Figure 4), despite the amputation surgery. While the EMG activity of their dorsiflexors and plantarflexors were consistent over multiple (29 to 72) gait cycles, the timing of this activation differed from that of non-amputees. Both subjects A1 and A3 exhibited dorsiflexion activity during loading response and swing, similar to that for non-amputees. Their plantarflexors, however, were relatively inactive during mid- and terminal stance.

Despite demonstrating independent muscle control during myotesting, the dorsiflexor and plantarflexor activity of subject A2 (and perhaps subject A3) exhibited substantial cross-talk during ambulation. Cross-talk of surface EMGs occurs due to EMG signal conduction, both radially and circumferentially, through the soft tissue bulk due to relative proximity of superficial/deep muscles and nearby muscle groups. In the intact lower limb, the dorsiflexors are in the anterior compartment; the plantarflexors are in the posterior compartment. After transtibial amputation, the location of the severed muscle groups and the relative location of the agonistantagonist muscle pairs may be altered due to the incident trauma and/or myoplasty to keep the muscles taut and provide distal padding. To minimize cross-talk between the ankle plantarflexors and dorsiflexors for these subjects, the potential electrode sites were selected such that minimal antagonist signal was observed during agonist contraction. The gains of the respective dorsiflexor and plantarflexors amplifiers were also set independently. For future lower extremity myoelectric control, independent myoactivity of respective agonistantagonist muscle pairs is likely necessary for proportional, dual-site control of an active ankle, but not for single site control.

The cross-talk observed between dorsiflexion and plantarflexion activity for subject $A 2$ is not unexpected as this subject had onsite traumatic amputation with several muscle surgeries post-amputation to provide good distal coverage of her residual limb. These surgeries resulted in anterior positioning of both the dorsiflexors and plantarflexors; this closer proximity increased the likelihood of EMG cross-talk between sites.

The latter gait trials in which the subjects were instructed to 'push off' during late stance were conducted to assess whether these subjects could consciously alter their plantarflexor activity, similar to that which occurred 
prior to amputation. If successful, such training might facilitate intuitive control of active ankle-foot prostheses in which the prosthetic motor replicates gastrocnemius activity and function during mid- to late-stance. Subjects $A 1$ and $A 2$ were able to alter their muscle activity during these latter gait trials. Subject $A 1$ was able to dramatically increase plantarflexor activity during mid-stance through pre-swing, despite only two to four gait cycles of instruction and training. Subject A2 was able to alter the timing of the relative plantarflexor/dorsiflexor activity, although the plantarflexor activity actually decreased during late stance. The plantarflexor and dorsiflexor activity and timing for subject A3 did not change between nominal and 'push off' trials. For all subjects, however, 'push off' training was minimal. Their relative plantarflexor activity during late stance may perhaps be increased with more extensive training.

\section{Conclusion}

These preliminary trials involving residual limb EMG activity during gait for three trans-tibial amputees indicate that: 1) surface EMG activity of the phantom ankle dorsiflexors and plantarflexors can be monitored during gait; 2) consistent EMG activity of the remnant dorsiflexors and plantarflexors is observed during gait up to 4 years post-amputation surgery for traumatic trans-tibial amputees; and 3) with minimal training, traumatic trans-tibial amputees may be able to alter their dorsiflexor and plantarflexor activity during gait. Based on these results, surface EMG control based on the remnant ankle plantar and dorsiflexors using upper extremity myoelectric control techniques therefore holds promise for future control of active ankle prosthetic components.

\section{Acknowledgements}

The authors acknowledge Hanger Prosthetics \& Orthotics, Inc. who provided the experimental sockets, gel liners, button electrodes, pre-amplifiers, and MyoBoy ${ }^{\circledR}$ EMG tester used in this research. They also gratefully acknowledge the support of Allison Hyngstrom, PhD who provided access to the Locomotor Neurorehabilitation Laboratory and Eric Walker, MS who assisted with the gait analyses.

\section{Funding}

This research received no specific grant from any funding agency in the public, commercial, or not-for-profit sectors.

\section{References}

1. $\quad \mathrm{Au}, \mathrm{S}, \mathrm{Weber}, \mathrm{F}, \mathrm{Herr}, \mathrm{H}$. Biomechanical design of a powered ankle-foot prosthesis. 10th International Conference on Rehabilitation Robotics, Netherlands, 2007: 298-303.

2. Au, S, Herr, H, Weber, J, Martinez-Villalpando, EC. Powered ankle-foot prosthesis for the improvement of amputee ambulation. IEEE EMBS - 29th Annual International Conference, Lyon, France, 2007: 3020-6.

3. Sup, F, Varol, HA, Mitchell, J, Withrow, T, Goldfarb, M. Design and control of an active electrical knee and ankle prosthesis. Biennial IEEE/RAS-EMBS International Conference of Biomedical Robotics and Mechatronics, Scottsdale, AZ, 2008: 523-8.

4. $\mathrm{Au}, \mathrm{S}$, Berniker, $\mathrm{M}, \mathrm{Herr}, \mathrm{H}$. Powered ankle-foot prosthesis to assist level-ground and stair-descent gaits. Neural Netw 2008; 21: 654-66.

5. Bergelin, B, Mattos, J, Wells, J, Voglewede, P. Concept through preliminary bench testing of a powered lower limb prosthetic device. J Mech Robotics 2010; 2(4): 41005-13.

6. Varol, HA, Sup, F, Goldfarb, M. Multiclass Real-Time Intent Recognition of a Powered Lower Limb Prosthesis. IEEE Trans Biomed Eng 2010; 57(3): 542-51.

7. Martin, J, Pollock, A, Hettinger, J. Microprocessor lower limb prosthetics: review of current state of the art. J Rehabil Res Develop 2010; 22(3): 183-93. 


\begin{tabular}{|c|c|}
\hline 8. & $\begin{array}{l}\text { Au, S, Bonato, P, Herr, H. EMG-position controlled system for an active ankle-foot prosthesis: an initial } \\
\text { experimental study. International Conference on Rehabilitation Robotics, Chicago, IL, 2005: 375- } \\
\text { 9. }\end{array}$ \\
\hline 9. & $\begin{array}{l}\text { Au, S, Berniker, M, Herr, H. Powered ankle-foot prosthesis to assist level-ground and stair-descent gaits. } \\
\text { Neural Networks 2008; 21(4): 654-66. }\end{array}$ \\
\hline 10. & $\begin{array}{l}\text { Zecca, M, Micera, S, Carrozza, MC. Control of multifunctional prosthetic hands by processing the } \\
\text { electromyographic signal. Crit Rev Biomed Eng } 2002 ; 30(4-6): 459-85 .\end{array}$ \\
\hline 11. & $\begin{array}{l}\text { Grimes, DL, Flowers, WC, Donath, M. Feasibility of an active control scheme for above knee prostheses. } \\
\text { ASME J Biomech Eng 1977; 99(4): 215-21. }\end{array}$ \\
\hline 12. & $\begin{array}{l}\text { Culham, EG, Peat, M, Newell, E. Below-knee amputation: a comparison of the effect of the SACH foot } \\
\text { and single axis foot on electromyographic patterns during locomotion. Prosthet Orthot Int 1986; } \\
\text { 10: } 15-22 \text {. }\end{array}$ \\
\hline 13. & $\begin{array}{l}\text { Isakov, E, Keren, O, Benjuya, N. Trans-tibial amputee gait: time-distance parameters and EMG activity. } \\
\text { Prosthet Orthot Int 2000; 24(3): 216-20. }\end{array}$ \\
\hline 14. & $\begin{array}{l}\text { Torburn, L, Perry, J, Ayyappa, E, Shanfield, SL. Below-knee amputee gait with dynamic elastic response } \\
\text { prosthetic feet: a pilot study. J Rehabil Res Dev 1990; 27(4): 369-84. }\end{array}$ \\
\hline 15. & Winter, DA, Sienko, SE. Biomechanics of below-knee amputee gait. J Biomechanics 1988; 21(5): 361-67. \\
\hline 16. & $\begin{array}{l}\text { Perry, J . Phases of gait. In: Gait analysis: normal and pathological function. Thorofare, NJ: SLACK } \\
\text { Inc., 1992: 9-18. }\end{array}$ \\
\hline 17. & $\begin{array}{l}\text { Perry, J . Dynamic electromyography. In: Gait analysis: normal and pathological function. Thorofare, } \\
\text { NJ: SLACK Inc., 1992: 381-412. }\end{array}$ \\
\hline
\end{tabular}

